\title{
Lidil
}

Revue de linguistique et de didactique des langues

$51 \mid 2015$

Acquisition du langage et phonologie

\section{Mariella Causa, Stéphanie Galligani et Monica Vlad (dir.), Formation et pratiques enseignantes en contextes pluriels}

Riveneuve Éditions, coll. « Actes académiques, série Langues et perspectives didactiques ", 2014, $413 \mathrm{p}$.

Nathalie Thamin

\section{CpenEdition}

\section{Journals}

Édition électronique

URL : http://journals.openedition.org/lidil/3792

DOI : 10.4000/lidil.3792

ISSN : 1960-6052

\section{Éditeur}

UGA Éditions/Université Grenoble Alpes

Édition imprimée

Date de publication : 30 mai 2015

Pagination : 245-247

ISBN : 978-2-84310-297-4

ISSN : $1146-6480$

\section{Référence électronique}

Nathalie Thamin, « Mariella Causa, Stéphanie Galligani et Monica Vlad (dir.), Formation et pratiques enseignantes en contextes pluriels », Lidil [En ligne], 51 | 2015, mis en ligne le 30 novembre 2016, consulté le 24 septembre 2020. URL : http://journals.openedition.org/lidil/3792 ; DOI : https://doi.org/ 10.4000/lidil.3792 
technicité, contrairement à la conférence donnée à l'université de Rouen en 1978 (p. 139), où les grilles sont justifiées selon des critères explicites d'analyse outillée de l'oral. La mise en grille est née comme réponse à l'impossibilité d'utiliser l'analyse grammaticale et logique de l'école pour rendre compte des textes oraux. Ensuite, en s'appuyant sur ses recherches, C. Blanche-Benveniste propose une simplification de la terminologie grammaticale et une progression dans la complexité syntaxique.

Dans le troisième chapitre, «La langue des élèves», les manuscrits et les articles montrent comment la rigueur de la description syntaxique des données de langue orales et écrites conduit à redéfinir la notion de compétence linguistique, intégrée dans l'enseignement et largement présente dans la société. L'analyse des corpus oraux et écrits de la langue des élèves prouve que, dans des situations de parodie, les élèves sont capables d'endosser ce que C. Blanche-Benveniste a appelé la «langue du dimanche». En effet, confrontés à des modèles linguistiques divers, les élèves savent adapter leur langage à la situation, parfois jusqu'à l'hypercorrection (manuscrit Parodies, 2002). À partir de ses analyses, C. Blanche-Benveniste intervient dans le débat éducatif et questionne les notions de compétence et de «handicap linguistique». Sa réflexion sur l'ancrage de la parole dans un contexte qui la surdétermine se poursuit avec la notion de genre que C. Blanche-Benveniste développe dans ses derniers travaux, notamment en référence à Biber.

Sandra Trevisi

LIDILEM et GRAFE (Groupe romand d'analyse du français enseigné,

FPSE, Université de Genève)

Mariella Causa, Stéphanie Galligani et Monica Vlad (dir.), Formation et pratiques enseignantes en contextes pluriels, Riveneuve Éditions, coll. «Actes académiques, série Langues et perspectives didactiques», 2014, $413 \mathrm{p}$.

Formation et pratiques enseignantes en contextes pluriels est l'un des derniers ouvrages en date de la collection récente intitulée «Actes académiques, série Langues et perspectives didactiques» publié chez Riveneuve éditions. Il se centre sur les questions de formation et de pratiques professionnelles des enseignants de langues, inscrites dans des contextes 
d'enseignement/apprentissage et de formation diversifiés (pour adultes, initiale et continue, universitaires) et résolument marqués par la pluralité : pluralité des contextes sociolinguistiques (français, roumain, catalan, canadien...), des langues présentes dans le répertoire linguistique des enseignants et des apprenants, pluralité des méthodologies, des répertoires didactiques enseignants (Cadet et Causa, 2005, p. 164 ${ }^{1}$ ) et des cultures éducatives.

Pour les directrices de publications en effet, l'objectif essentiel des contributions vise à interroger la discipline «des langues» dans sa dynamique, en appréhendant les notions de changement, de besoins en formation et de contextes. La formation y est conçue comme «espace de construction de compétences et de pratiques enseignantes situées, de professionnalisation, mais aussi d'échanges et de participations collaboratifs au projet formatif» (Causa, Galligani et Vlad, introduction, p. 9). La dimension de co-construction des «valeurs et des pratiques de transmission et de prise en charge des langues et des cultures permettant aux (futurs) enseignants de s'adapter au changement, de répondre aux besoins de diversification et d'œuvrer en contextes pluriels» (p. 11) y prend une place fondamentale. Dans cette optique, les contributions proposent des réflexions d'ordres multiples (théoriques, didactiques, épistémologiques, méthodologiques) dont un certain nombre s'inscrit directement dans des recherches-actions-formations $\left(\mathrm{RAF}^{2}\right)$ où l'implication des «formés partenaires engagés de la recherche» est partie intégrante de la formation et pratique enseignante et où les chercheurs s'engagent dans l'action formative.

Les 24 contributions proposées en font un ouvrage dense qui s'organise en trois parties cohérentes autour des actes de professionnaliser (partie 1), d'éduquer au plurilinguisme et à l'interculturalité (partie 2), et enfin de développer la réflexivité (partie 3). Chacune d'elles est constituée de cinq à sept articles et complétée par une section de deux articles intitulée «en complément», dont le rôle est d'élargir les perspectives de réflexion liées au contexte contemporain nourrissant la didactique des

1. L. Cadet et M. Causa (2005), «Cultures éducative(s) et construction d'un répertoire didactique en formation initiale», dans J.-C. Beacco, J.-L. Chiss, F. Cicurel et D. Véronique (dir.), Les cultures éducatives et linguistiques dans l'enseignement des langues, Paris, PUF, p. 159-181.

2. Méthodologie adoptée et développée par le groupe FICEL (Formation initiale et continue des enseignants de langues) du laboratoire DILTEC (Didactique des langues, des textes et des cultures), EA2288, Université Sorbonne Nouvelle - Paris 3. 
langues. Y sont considérés les composantes, les enjeux et les défis de l'éducation plurilingue et interculturelle, l'importance des approches biographiques et des approches méthodologiques communicative et actionnelle en contextes plurilingues (Castelloti, Galligani, Simon...), la question des langues familiales (Goletto) et de leur articulation transversale avec les autres langues (de scolarisation, étrangères...) dans les enseignements linguistiques et disciplinaires (Cavalli, Forlot).

La grande richesse de l'ouvrage est aussi de proposer des angles de vue variés et complémentaires sur l'objet traité : apport de la linguistique saussurienne à la didactique des langues (Bulea); apport des outils formatifs comme la notion de genre discursif, l'analyse du discours (Claudel), le texte de théâtre (Rollinat-Vasseur), l'approche grammaticale (Bruley), les outils biographiques; réflexion sur les compétences professionnelles enseignantes trop rarement traitées par ailleurs comme la dimension éthique (Bretegnier) ou encore le développement personnel (Aguilar Río), mais aussi les savoirs enseignants (Causa); réflexions méthodologiques à partir de l'observation participante (Goletto), de la trame méthodique repère (Laurens), des Représentations Croyances Savoir (Cambra-Giné, Birello)...

La notion de réflexivité, héritée des approches biographiques en sciences de l'éducation, est mise en relief dans le dernier chapitre. L'article introductif de Bretegnier propose une synthèse théorique très utile où la réflexivité est décrite comme macro-compétence dynamique ( «savoir agir en contexte et en relation»), mais aussi comme posture, rapport au monde, au savoir, à la complexité (Perrenoud, 2005, p. 313, cité par Bretegnier, p. 275), impliquant une «démarche analytique contextualisée ». Elle est envisagée comme compétence inhérente au processus de professionnalisation des enseignants, qu'elle soit professionnelle/enseignante, éthique, grammaticale, linguistique.

L'ensemble de l'ouvrage vise finalement à donner aux lecteurs avertis (étudiants, enseignants, enseignants-chercheurs, formateurs) des pistes pertinentes de réflexion pour œuvrer au développement de l'identité professionnelle enseignante.

Nathalie Thamin

Université de Franche-Comté, Besançon

3. P. Perrenoud (2005), «Assumer une identité réflexive», Éducateur, n ${ }^{\circ}$, p. 30-33. 